\title{
GROUP LAWS IMPLYING VIRTUAL NILPOTENCE
}

\author{
R. G. BURNS and YURI MEDVEDEV
}

(Received 31 October 2001; revised 24 February 2002)

Communicated by R. B. Howlett

\begin{abstract}
If $w \equiv 1$ is a group law implying virtual nilpotence in every finitely generated metabelian group satisfying it, then it implies virtual nilpotence for the finitely generated groups of a large class $\mathscr{S}$ of groups including all residually or locally soluble-or-finite groups. In fact the groups of $\mathscr{S}$ satisfying such a law are all nilpotent-by-finite exponent where the nilpotency class and exponent in question are both bounded above in terms of the length of $w$ alone. This yields a dichotomy for words. Finally, if the law $w \equiv 1$ satisfies a certain additional condition-obtaining in particular for any monoidal or Engel law-then the conclusion extends to the much larger class consisting of all 'locally graded' groups.
\end{abstract}

2000 Mathematics subject classification: primary 20F19, 20E10, 20F45.

\section{Introduction}

Let $F$ denote the free group on $X=\left\{x_{1}, x_{2}, \ldots\right\}$. A law (or identical relation) of a group $G$ is an identity $w \equiv 1$ where $w$ is a word from $F$, valid under every substitution $X \rightarrow G$.

The following two types of group laws are of particular interest: A positive (or monoidal) law of a group $G$ is a nontrivial identity of the form $u \equiv v$ (or $u v^{-1} \equiv 1$ ) where $u, v$ are positive words in $F$, that is, do not involve any of the inverses of the $x_{i}$. The $n$-Engel law is the law $[\cdots[[x, y], y], \ldots, y] \equiv 1$, where $y$ appears $n$ times, $x:=x_{1}, y:=x_{2}$, and $[a, b]:=a^{-1} b^{-1} a b$, the commutator of elements $a, b$ of an arbitrary group $G$. (We shall use the 'left-normed' convention, defining inductively $\left[a_{1}, \ldots, a_{n}\right]:=\left[\left[a_{1}, \ldots, a_{n-1}\right], a_{n}\right]$. The $n$-Engel law then takes the form $[x, y, y, \ldots, y] \equiv 1$, which is often further abbreviated to $\left[x,{ }_{n} y\right] \equiv 1$.)

(C) 2003 Australian Mathematical Society 1446-7887/03\$A2.00+0.00 
It is well known that a nilpotent-by-finite exponent group, that is, one with a nilpotent normal subgroup with quotient of finite exponent, satisfies a monoidal law $([11,12])$, and it is immediate that a nilpotent group satisfies the $n$-Engel law for some $n$. The converse of neither of these statements is true in general: there exists a 2-generator group which is not nilpotent-by-finite exponent yet satisfies a monoidal law [14], and it is relatively easy to find a group (albeit infinitely generated) which is $n$-Engel for some $n$ yet not nilpotent (see for example [8, page 132] or [17, page 362, Exercise 1]).

We are interested here in the much-studied question as to the extent to which, that is, for how wide a class of groups, some versions of the converse statements do after all hold, and also in characterizing those laws which behave in this respect like monoidal and Engel laws, that is, imply 'virtual nilpotence' for the same large class of groups.

Our results are as follows. We write $\mathscr{S}$ for the class of groups obtained from the class of all groups that are soluble-by-(locally finite of finite exponent) by closing under the operators $L$ and $R$, where for any group-theoretic class $\mathscr{X}, L \mathscr{X}$ denotes the class of all groups locally in $\mathscr{X}$ and $R \mathscr{X}$ the class of groups residually in $\mathscr{X}$ (see [16, Section 1.1]). (Thus $\mathscr{S}$ contains in particular all residually finite or soluble groups, and all locally finite or soluble groups.) We also write $\hat{\mathfrak{B}}_{e}$ for the variety consisting of all locally finite groups of exponent dividing $e$ (the hat distinguishing this variety from the variety $\mathfrak{B}_{e}$ of all groups of exponent dividing $e$ ), and, as usual, $\mathfrak{N}_{c}$ for the variety of all nilpotent groups of class $\leq c$. (Note that the fact that the class $\hat{\mathfrak{B}}_{\varepsilon}$ is actually a variety, is a consequence of Zelmanov's solution of the restricted Burnside problem $[24,23]$.) Our main result gives a necessary and sufficient condition for a law $w \equiv 1$ to single out from the class $\mathscr{S}$ just the nilpotent-by-finite exponent groups, furnishing in addition the information that the nilpotency class and exponent in question are bounded above solely in terms of the length of $w$.

THEOREM A (Compare [1, Theorem]). Let $w$ be any word in $F$ with the property that every finitely generated metabelian group satisfying the law $w \equiv 1$ is necessarily nilpotent-by-finite. Then there exist positive integers $c=c(N), e=e(N)$ depending only on the length $N$ of $w$ such that the groups in the class $\mathscr{S}$ satisfying $w \equiv 1$ are just those in the product variety $\mathfrak{N}_{c} \hat{\mathfrak{B}}_{e}$.

That a monoidal law (in the form $u v^{-1} \equiv 1$, where $u, v$ are positive words) satisfies the hypothesis of Theorem A will emerge in the course of the proof. (This also follows from for instance Point [15].) That the $n$-Engel law does so follows from Gruenberg's result [5] that a finitely generated soluble Engel group is nilpotent, and therefore satisfies a monoidal law.

Theorem A is stronger than related results (for example of Point [15]) in that the nilpotency class $c$ figuring in the conclusion, as well as the exponent $e$, depend only on the length of $w$. It is this that allows the conclusion to be extended to the full class 
$\mathscr{S}$, as opposed to just finitely generated soluble-by-finite groups, and in Theorem B below, where an additional condition is imposed on $w$, to the very large class of 'locally graded' groups.

As a consequence of Theorem A and a result of Groves [4], we infer the following

DICHOTOMY THEOREM (for words of $F$ ). Let $w \in F$ be an arbitrary word. Exactly one of the following two possibilities occurs:

(i) every group in $\mathscr{S}$ satisfying the law $w \equiv 1$ is nilpotent-by-finite exponent;

(ii) for some $n \geq 2, w \equiv 1$ is a law in the restricted wreath product $C_{n} 2 C$, where $C_{n}, C$ denote the cyclic group of order $n$ and the infinite cyclic group respectively.

(To see this, suppose that for every prime $p, w \equiv 1$ is not a law in $C_{p} \geq C$, that is, that (ii) fails to hold. Since $C_{p}<C$ generates the product variety $\mathfrak{A}_{p} \mathfrak{A}$ of the variety of all abelian groups of exponent $p$ by the variety of all abelian groups (see for example [13, Corollary 22.44]), it follows that for any soluble (in particular) variety $\mathfrak{V}$ satisfying the law $w \equiv 1$, we have $\mathfrak{A}_{p} \mathfrak{A} \nsubseteq \mathfrak{V}$ for all primes $p$, whence by the dichotomy established by Groves [4, Theorem A (ii)], $\mathfrak{V} \subseteq \mathfrak{N}_{c_{1}} \hat{\mathfrak{B}}_{e_{1}}$ for some $c_{1}, e_{1}$. Hence certainly every metabelian group satisfying $w \equiv 1$ is nilpotent-by-finite exponent, so that $w$ satisfies the assumption of Theorem $A$, and thence the alternative statement (i).)

A partially related dichotomy is that of Rosenblatt [18] according to which any group $G$ not containing the free monoid $M_{2}$ on two generators is restrained, that is, for every pair of elements $a, b$ of $G$, the subgroup $\langle a\rangle^{(b)}$ is finitely generated (or, equivalently, every finitely generated subgroup $H$ has finitely generated commutator subgroup $[H, H]$ ). (It follows easily from this that for every prime $p$, the wreath product $C_{p} ; C$ embeds $M_{2}$.) A negative answer to the following question would enable the replacement of the class $\mathscr{S}$ in Theorem A and the Dichotomy Theorem by a much larger class-perhaps even that of 'locally graded' groups. (A group is locally graded if every non-trivial finitely generated subgroup has a proper subgroup of finite index.) Does there exist a group $G$ having a minimal normal subgroup $H$ which is infinitely generated, locally finite and of finite exponent, with $G / H$ infinite cyclic, such that $G$ obeys a law $w \equiv 1$ satisfying the assumption of Theorem $\mathrm{A}$, that is, not holding in any $C_{p}$ ? $C$ ? It can be shown that such a group could not be restrained, and would therefore have to embed $M_{2}$, in view of Rosenblatt's result. It would thus furnish an example showing that Theorem A cannot be extended to the class of locally graded groups unless $w$ satisfies some additional condition.

Among the laws $w \equiv 1$ satisfying the hypothesis of Theorem A, there are manyincluding monoidal laws and the Engel laws-that do satisfy a condition allowing the extension of Theorem A to the class of locally graded groups. It is well known (see $[2,3,5,9,15])$ that if a group $G$ satisfies a monoidal law or an Engel law then $G$ is uniformly restrained, that is, there is a positive integer $k$ such that for all $a, b \in G$ 
the subgroup $\langle a\rangle^{(b)}$ can be generated by $\leq k$ elements. By Kim and Rhemtulla [9], a finitely generated, uniformly restrained, locally graded group is polycyclic-by-finite. Hence applying Theorem A we obtain immediately

THEOREM B. Let $w \equiv 1$ be a group law with the following two properties:

(i) every finitely generated metabelian group satisfying $w \equiv 1$ is nilpotent-byfinite;

(ii) every group satisfying $w \equiv 1$ is uniformly restrained.

Then there exist positive integers $c=c(N), e=e(N)$ depending only on the length $N$ of the word $w$, such that every locally graded group satisfying the law $w \equiv 1$ lies in $\mathfrak{N}_{c} \hat{\mathfrak{B}}_{\boldsymbol{e}}$.

A natural question here is whether the qualifier 'uniformly' can be omitted from condition (ii).

COROLlaRY 1 (Compare [2, Theorem B]). For any monoidal law $u \equiv v$, the locally graded groups satisfying that law all lie in $\mathfrak{N}_{c} \hat{\mathfrak{B}}_{e}$ for some $c$, e depending only on the larger of the lengths of $u, v$.

The analogue for Engel laws can be improved to the following result (see [3]), which implies, but is more detailed than, the result of Kim and Rhemtulla [9] that locally graded $n$-Engel groups are locally nilpotent.

COROLLARY 2 (Compare [3, Theorem]). For each positive integer $n$ there existpositive integers $c=c(n), e=e(n)$ depending only on $n$ such that every locally graded $n$-Engel group lies in $\mathfrak{N}_{c} \hat{\mathfrak{B}}_{e} \cap \hat{\mathfrak{B}}_{e} \mathfrak{N}_{c}$.

REMARKS. 1. The class of locally graded groups, which contains the class $\mathscr{C}$ of $[2,3]$, is large in - for instance - the sense that it contains nearly all the groups encountered in standard textbooks on group theory (for example $[8,17]$ ), or that it consists of the largest class of groups 'constructible' from the class of finite groups by closing under most standard and some not-so-standard group-theoretic operations. (A. Rhemtulla has mentioned to us the possibility that the class of locally graded groups may coincide with that of groups with a generalized series (in one sense or another-see for example [8, page 160], [16, page 9]) with finite factors.)

2. There would seem to be considerable overlap between the main result of the present paper and that of Sarah Black [1] (and also those of [2, 3], which it generalizes). In fact [1] gives in essence a characterization of words $w$ yielding the conclusion of our Theorem A, but in terms of the form of $w$ itself. However the results of $[1,2,3]$ depended ultimately on a lemma of Shalev [20, Lemma 3.2, Case 2] which is not quite valid as stated. (For the details, see the Remark in Section 2.3.2 below.) Thus 
apart from the improvements claimed for the results of the present paper over those of $[1,2,3]$, it is also justified by the need to provide arguments based on the corrected version of Shalev's lemma. Nevertheless the arguments we use, although mostly self-contained, are to a considerable extent adapted from those of Shalev [20], as well as $[1,2,15]$.

The layout of the proof of Theorem A is as follows: In Section 2 we establish the finite case by means of a succession of reductions. The proof of the theorem is then completed in Section 3.

We are grateful to Olga Macedońska for helpful comments.

\section{Proof of Theorem A for finite groups}

Thus in this section we shall prove that if $w \equiv 1$ is a law with the property that every finitely generated metabelian group satisfying it is nilpotent-by-finite, then there exist positive integers $c=c(N), e=e(N)$ depending only on the length $N$ of $w$ such that for every finite group $G$ satisfying $w \equiv 1$ we have $G^{e}$ (the subgroup generated by all $e$-th powers of elements of $G$ ) nilpotent of class $\leq c$.

Write $F_{2}$ for the free group of rank 2 generated by $x, y$. We shall need the following

LEMMA 1. Let $w$ be as above (that is, as in Theorem A). Then the law $w \equiv 1$ has as a consequence a 2-variable law of the form

$$
x\left(x^{s_{1}}\right)^{y^{t_{1}}} \cdots\left(x^{s_{k}}\right)^{y^{\prime k}}=c,
$$

where $k \geq 1$, the $s_{i}$ and $t_{i}$ are nonzero integers with $0>t_{1}>\cdots>t_{k}$, and $c$ is a product of commutators in elements of the form $x^{y^{\alpha}}:=y^{-\alpha} x y^{\alpha}, \alpha \in \mathbb{Z}$.

Proof. Denote by $W_{2}$ the verbal subgroup of $F_{2}$ determined by $w$. Since $\hat{F}_{2}:=$ $F_{2} / F_{2}^{(2)} W_{2}$ is a finitely generated metabelian group satisfying the law $w \equiv 1$, it follows from our basic assumption concerning $w$ that $\hat{F}_{2}$ is nilpotent-by-finite, and therefore $([11,12])$ satisfies a non-trivial monoidal (here actually semigroup) law

$$
u \equiv v,
$$

where $u, v$ are non-trivial positive words in $x$ and $y$ of equal exponent sums on each of $x$ and $y$. We may, by interchanging the variables $x$ and $y$ and cancelling, if necessary, suppose that $u$ begins with $x$ and $v$ with $y$, and that $u$ and $v$ end in different symbols from $\{x, y\}$. Furthermore if $u$ does not have $x y$ as initial segment, then by applying the change of variables $x \rightarrow x y, y \rightarrow y$, we can ensure that it does. 
Assuming this done, we lift the law (2) to $F_{2} / W_{2}$, obtaining a relation in $F_{2} / W_{2}$ of the form

$$
\bar{u} \bar{v}^{-1}=\bar{d},
$$

where $d \in F_{2}^{(2)}$, and the bars indicate elements in $F_{2} / W_{2}$. By virtue of the relative freeness of $F_{2} / W_{2}$, the relation (3) will then define a law $\left(u v^{-1} \equiv d\right)$ in any group satisfying $w \equiv 1$, that is, represents a 2-variable consequence of $w \equiv 1$.

Note that the word $u v^{-1}$ is reduced as written (since $u, v$ end in different symbols from $\{x, y\})$ and has exponent sums 0 on each of $x, y$. We now rewrite this word as a product of elements of the form $\left(x^{s}\right)^{y^{t}}$, starting on the left. Since $u$ has $x y$ as initial segment the first such element will be simply $x$. It is not difficult to see that since $v^{-1}$ ends in $y^{-1}$, all other factors of the required form $\left(x^{5}\right)^{y^{t}}$ will have $t<0$. (To illustrate, consider $u=x y^{2} x^{2} y^{3} x$ and $v=y^{2} x^{2} y x^{2} y^{2}$. Here

$$
\left.u v^{-1}=x y^{2} x^{2} y^{3} x y^{-2} x^{-2} y^{-1} x^{-2} y^{-2}=x\left(x^{2}\right)^{y^{-2}} x^{y^{-5}}\left(x^{-2}\right)^{y^{-3}}\left(x^{-2}\right)^{y^{-2}} .\right)
$$

Then by collecting the factors $\left(x^{s}\right)^{y^{t}}$ of $u v^{-1}$ modulo $\left[\langle x\rangle^{F_{2}},\langle x\rangle^{F_{2}}\right]$, the law $u v^{-1} \equiv d$ implied by (3) becomes $u v^{-1}=x\left(x^{s_{1}}\right)^{y^{t_{1}}} \cdots\left(x^{s_{k}}\right)^{y^{t_{k}}} \gamma \equiv d$, where $k>0,0>t_{1}>$ $\cdots>t_{k}$, the $s_{i}$ are all nonzero, and $\gamma \in\left[\langle x\rangle^{F_{2}},\langle x\rangle^{F_{2}}\right]$. Since $\left[\langle x\rangle^{F_{2}},\langle x\rangle^{F_{2}}\right]>F_{2}^{(2)}$, we have $d \gamma^{-1}=: c \in\left[\langle x\rangle^{F_{2}},\langle x\rangle^{F_{2}}\right]$, and we have arrived at a consequence of the law $w \equiv 1$ of the desired form (1).

COROLlaRY 3. For each $N>0$ there exist positive integers $L=L(N), R=R(N)$ depending only on $N$, such that each word $w$ of length $\leq N$ satisfying the assumption of Theorem A has a 2-variable consequence of the form (1), namely

$$
x\left(x^{s_{1}}\right)^{y_{1}} \cdots\left(x^{s_{k}}\right)^{y_{k}^{t_{k}}} \equiv c
$$

where $k>0$, the $s_{i}, t_{i}$ are nonzero integers, $0>t_{1}>\cdots>t_{k}, c$ is a product of commutators in elements of the form $x^{y^{\alpha}}, \alpha \in \mathbb{Z}$, and also $\left|t_{k}\right| \leq L$ and $|\alpha| \leq R$ for all integers $\alpha$ such that $x^{ \pm y^{\alpha}}$ figures as an entry in a commutator occurring as a factor of $c$.

(This is immediate from Lemma 1 and the fact that there are only finitely many such words $w$ of length $\leq N$ (in, say, $x_{1}, \ldots, x_{N}$ ).)

We now begin the proof of Theorem A for arbitrary finite $\mathrm{G}$ satisfying a law $w \equiv 1$ obeying the assumption of that theorem. The proof involves a sequence of reductions.

2.1. Reduction to the finite soluble case. We shall show that there exists a positive integer $e_{1}$ depending only on the length $N$ of $w$ such that $G^{e_{1}}$ is soluble.

Let $A / B$ be any non-abelian chief factor of the finite group $G$. (If there are no such chief factors then $G$ is soluble and we may take $e_{1}=1$.) It is well known that $A / B$ 
decomposes as a direct product $S_{1} \times S_{2} \times \cdots S_{l}$ of finite non-abelian simple groups all isomorphic to the same simple group $S$, and that each element $g$ of $G$ acts by conjugation on $A / B$ in such a way as to permute the $S_{i}$; write $\pi_{g}$ for the permutation of $\left\{S_{1}, \ldots, S_{l}\right\}$ induced by conjugation by $g$.

Write $c=c(x, y)$ for the right-hand side of the law (4), a consequence of $w \equiv 1$. We first show that for each $g \in G$ if any orbit under the above action of $g$ on $\left\{S_{1}, \ldots, S_{l}\right\}$ has size exceeding $2 R$, then for every member $S_{r}$ of that orbit we have

$$
c(h B, g B)=1 \text { in } A / B \text { for all } h B \in S_{r} .
$$

This is seen as follows. Suppose $c(x, y) \neq 1$ (otherwise there is nothing to prove). Let $x^{y^{\alpha_{1}}}, x^{y^{\alpha_{2}}}$ be distinct entries in (possibly coinciding) commutator factors of $c$. We cannot have $S_{r}^{g^{\alpha_{1}}}=S_{r}^{g^{\alpha_{2}}}$, since this would imply that $S_{r}^{g^{\alpha_{1}-a_{2}}}=S_{r}$, and the orbit containing $S_{r}$ would then have size at most $\left|\alpha_{1}-\alpha_{2}\right| \leq 2 R$ (see Corollary 3), contrary to our assumption. Hence $S_{r}^{g^{\alpha_{1}}}=S_{i} \neq S_{j}=S_{r}^{g^{\alpha_{2}}}$, yielding $\left[(h B)^{g^{\alpha_{1}}},(h B)^{g^{\alpha_{2}}}\right]=1$ for all $h B \in S_{r}$, that is, all elements of the form $(h B)^{g^{\alpha}}$ where $h B \in S_{r}$ and $x^{y^{\alpha}}$ is an entry in $c$, commute, so that $c(h B, g B)=1$ (in $G / B$ ) for all $g \in G, h B \in S_{r}$, as claimed.

Suppose now that $g \in G$ and $r \in\{1, \ldots, l\}$ are such that $c(h B, g B)=1$ for all $h B \in S_{r}$. Since (4) is a law in $G$, it follows that then

$$
h\left(h^{s_{1}}\right)^{g^{t_{1}}} \cdots\left(h^{s_{k}}\right)^{g^{t_{k}}} \equiv 1 \bmod B \text { for all } h B \in S_{r},
$$

where, as in (4), $k \geq 1,0>t_{1}>\cdots>t_{k}$, and the $s_{i}$ are all nonzero. Choose $h B \in S_{r}$ nontrivial (that is, $h B \neq B$ ); then since the cosets $\left(h^{s_{i}}\right)^{g_{i}} B$ belong to various of the direct factors $S_{1}, \ldots, S_{l}$ of $A / B$, in order for (5) to hold we must have for at least one $j \in\{1, \ldots, k\}$ that also $\left(h^{s_{j}}\right)^{g^{t_{j}} B} B$ is a (non-trivial) element of $S_{r}$, so that $S_{r}^{g^{t_{j}}}=S_{r}$ for this $j$. Hence in this situation the size of the orbit containing $S_{r}$ under the action of $\pi_{g}$ is bounded above by $\left|t_{k}\right|$, and therefore by the number $L$ of Corollary 3 .

We have thus established the following assertion: For each $g \in G$, every orbit of the action of $g$ by conjugation on $\left\{S_{1}, \ldots, S_{l}\right\}$, the set of simple direct factors of an arbitrary non-abelian chief factor $A / B$ of $G$, has size bounded above by $M:=$ $\max \{L, 2 R\}$, which depends only on the length $N$ of $w$.

It follows immediately that for every simple direct factor $S$ of every non-abelian chief factor of $G$ we have

$$
G^{M !} \leq N_{G}(S)
$$

By Jones [7] only the variety of all groups contains infinitely many pairwise nonisomorphic finite non-abelian simple groups. Hence in particular there are only a finite number of pairwise non-isomorphic groups that can occur as composition factors of groups satisfying any nontrivial law $\hat{w} \equiv 1$ of length $\leq N$ having the same property as $w \equiv 1$. Taking $K$ to be the largest of the orders of these simple groups, it follows 
that for every simple direct factor $S$ of every non-abelian chief factor of $G$ we have that $|\operatorname{Aut}(S)|$ divides $K$ !. From this and (6) we infer immediately that for any finite group $G$ satisfying $w \equiv 1$ we have $G^{M ! K !} \leq C_{G}(A / B)$ for every non-abelian chief factor $A / B$ of $G$, whence

$$
G^{M ! K !} \leq \bigcap C_{G}(A / B),
$$

where the intersection is taken over all non-abelian chief factors $A / B$ of $G$. It is not difficult to show that the intersection in (7) is a soluble subgroup of $G$, whence the desired conclusion with $e_{1}:=M ! K !$.

2.2. Reduction to the finite nilpotent case. By the previous reduction, we may now assume that our finite group $G$ satisfying the law $w \equiv 1$ is soluble. We shall now show that there is a positive integer $e_{2}$ depending only on the length $N$ of $w$ such that $G^{e_{2}}$ is nilpotent.

Let $G=G_{0} \triangleright G_{1} \triangleright \cdots \triangleright G_{r}=\{1\}$ be a chief series for $G$. Since $G$ is finite soluble, each chief factor $G_{i} / G_{i+1}$ is finite elementary abelian. Let $g$ be any element of $G$; then conjugation by $g$ induces an automorphism of each chief factor $G_{i} / G_{i+1}$. In the usual way we may consider $G_{i} / G_{i+1}$ as an (additively written) vector space $V_{i}$ over $\mathbb{Z}_{p}$ (where $p$ is the exponent of $G_{i} / G_{i+1}$ ) on which conjugation by $g$ induces an invertible linear transformation $T_{g}$. Now the subgroup $H / G_{i+1}$ of $G / G_{i+1}$ generated by $g G_{i+1}$ together with $G_{i} / G_{i+1}$ satisfies the law $w \equiv 1$ since $G$ does, and therefore also the 2-variable law (4). Replacing in that law $y$ by $\bar{g}^{l}:=g^{l} G_{i+1}$, for each $l=1,2, \ldots$ in turn, and $x$ by $\bar{h}:=h G_{i+1}$ for any $h \in G_{i}$, we obtain

$$
\bar{h}\left(\bar{h}^{s_{1}}\right)^{\bar{g}^{-t_{1}}} \cdots\left(\bar{h}^{s_{k}}\right)^{\bar{g}^{-t_{k}}}=1 \text { for all } h \in G_{i} \text {, and each } l=1,2, \ldots,
$$

since the right-hand side $c(x, y)$ of the law (4) becomes $c\left(\bar{h}, \bar{g}^{l}\right)=1$. In the additive notation of the vector space $V_{i}$, equation (8) translates into

$$
\left(I+s_{1} T_{g}^{l_{1}}+s_{2} T_{g}^{l l_{2}}+\cdots+s_{k} T_{g}^{l t_{k}}\right) V_{i}=\{0\} .
$$

Thus the linear transformations $T_{g}^{-l}, l=1,2, \ldots$, all satisfy the non-constant polynomial

$$
f(z):=1+s_{1} z^{-t_{1}}+s_{2} z^{-t_{2}}+\cdots+s_{k} z^{-t_{k}} \in \mathbb{Z}_{p}[z]
$$

At this juncture we need the following

LEMMA 2 (Compare [19, Lemma 3.3]). Let $K$ be any field, and $f(z) \in K[z]$ any polynomial over $K$ of degree $d>0$ with nonzero constant term. If $l$ is the ideal in $K[z]$ generated by $f(z), f\left(z^{2}\right), \ldots, f\left(z^{d+1}\right)$, then there exist positive integers $q, m$ bounded above by a function of $d$ only, such that $\left(z^{m}-1\right)^{q} \in I$. 
PROOF. Note that the ring $K[z]$ is a principal ideal domain and so of course also enjoys unique factorization. Let $g(z)$ be a generator of $I$, and let $p_{1}, \ldots, p_{t}$ be the distinct prime polynomials occurring as factors of $g(z)$. Observe that since $f(z)$ has non-zero constant term, so also do all of the $p_{i}$, and also that since $\operatorname{deg} g \leq \operatorname{deg} f=d$, we have $\operatorname{deg} p_{i} \leq d$ for all $i$, and $t \leq d$. Write $P_{i}:=\left(p_{i}\right)$, the ideal generated by $p_{i}, i=1, \ldots, t$, and consider the fields $F_{i}:=K[z] / P_{i}$. Regarded as having its coefficients in $F_{i}$, the polynomial $f(u)$ has roots $z+P_{i}, z^{2}+P_{i}, \ldots, z^{d+1}+P_{i}$, since $P_{i} \supseteq I$. Since $\operatorname{deg} f=d$, it follows that $z^{r_{i}} \equiv z^{s_{i}} \bmod P_{i}$ for some $0<r_{i}<s_{i} \leq$ $d+1$. Now $z \notin P_{i}$ since the $p_{i}$ all have non-zero constant term. Hence $z^{m_{i}} \equiv 1$ $\bmod P_{i}$ where $0<m_{i}:=s_{i}-r_{i} \leq d$. Writing $m:=m_{1} m_{2} \cdots m_{t}$, we infer that

$$
z^{m} \equiv 1 \quad\left(\bmod p_{1} p_{2} \cdots p_{t}\right)
$$

(Note that since $m_{i} \leq d, t \leq d$, we have $m \leq d^{d}$.) Let $q$ be largest such that $p_{i}^{q}$ is a factor of $g(z)$ for some $i$. (Observe that since deg $g \leq d$, we have $q \leq d$.) Then $\left(p_{1} \cdots p_{t}\right)^{q} \in(g(z))=I$, whence $\left(z^{m}-1\right)^{q} \in I$, as required.

We now return to our reduction argument. By the lemma just proved, applied to the polynomial $f(z)$ in (9), since $T_{g}^{-1}$ satisfies $f(z)=0, f\left(z^{2}\right)=0, \ldots$, we infer the existence of positive integers $m, q$ bounded above by a function of $t_{k}$ only, such that

$$
\left(T_{g}^{-m}-1\right)^{q}=0 .
$$

In terms of the multiplication in $G$, since $g$ is arbitrary this translates into:

$$
[h, \underbrace{g^{m}, \ldots, g^{m}}_{q}] \in G_{i+1} \text { for all } h \in G_{i}, g \in G .
$$

Since $i$ also was arbitrary, we have

$$
[h_{1}, \underbrace{g^{m}, \ldots, g^{m}}_{q}] \in G_{i+2} \text { for all } h_{1} \in G_{i+1}, g \in G .
$$

In particular putting $h_{1}=[h, \underbrace{g^{m}, \ldots, g^{m}}_{q}]$, we obtain

$$
[h, \underbrace{g^{m}, \ldots, g^{m}}_{2 q}] \in G_{i+2},
$$

and so on. Thus by induction we conclude that for all $g, h \in G$,

$$
[h, \underbrace{g^{m}, \ldots, g^{m}}_{r q}]=1
$$


that is, for every $g \in G, g^{m}$ is an Engel element (right Engel, in the terminology of [6]) of $G$. By [6, Lemma 6.13] a finite soluble group generated by Engel elements is nilpotent; hence $G^{m}$ is nilpotent.

Since $m$ is bounded above by a function of $\left|t_{k}\right|$ only, by arguing as in Corollary 3 we can show that $m$ is bounded above by a number $B$ say, depending only on $N$, the length of $w$, and we may then take $e_{2}:=B$ !.

2.3. The finite nilpotent case. In view of the above reductions, we may now assume that our finite group $G$ satisfying the law $w \equiv 1$ is nilpotent; in fact, since a finite nilpotent group is the direct product of its Sylow subgroups, we may assume that $G$ is a finite $p$-group for some prime $p$.

The proof of Theorem A in this case splits into two subcases.

2.3.1. Subcase: $p$ sufficiently large. This subcase concerns primes $p \geq T$ where $T=T(N)$ is a positive integer depending only on $N$, to be defined in the course of our argument. We begin with

LEMMA 3. As before let $F$ denote the free group with free basis $\left\{x_{1}, x_{2}, \ldots\right\}$. A law $w \equiv 1$ satisfying the assumption of Theorem A has as a consequence a law of the form

$$
\left[x_{1}^{\hat{e}}, \ldots, x_{\hat{c}}^{\hat{e}}\right] \equiv \gamma_{1} \gamma_{2} \cdots \gamma_{s}
$$

where each $\gamma_{i}$ is of the form $\left[\mu_{i}, v_{i}\right]$ with $\mu_{i}, v_{i}$ commutators in $x_{1}, \ldots, x_{\hat{c}}$ of weights $\geq 2$, and where for every $j=1, \ldots, \hat{c}$, either $x_{j}$ or $x_{j}^{-1}$ is an entry in $\mu_{i}$ or $v_{i}$ (so that each $\gamma_{i}$ has $x_{j}$ or $x_{j}^{-1}$ as an entry for all $\left.j=i, \ldots, \hat{c}\right)$.

Proof. Let $W$ denote the verbal subgroup of $F$ generated by $w$. Then $\bar{F}:=$ $F / W F^{(2)}$ is a metabelian group satisfying the law $w \equiv 1$. Since the wreath product $C_{p_{1}}<C$ of a cyclic group of prime order $p_{\mathrm{I}}$ with an infinite cycle, is finitely generated and metabelian but not nilpotent-by-finite, it cannot satisfy the law $w \equiv 1$. Hence in view of the fact that $C_{p_{1}}<C$ generates the product variety $\mathfrak{A}_{p_{1}} \mathfrak{A}$, we infer that $\mathfrak{A}_{p} \mathfrak{A} \nsubseteq \operatorname{var} \bar{F}$. Hence by [4, Theorem A (ii)] var $\bar{F}$ is contained in $\mathfrak{N}_{\hat{c}} \hat{\mathfrak{B}}_{\hat{e}}$ for some $\hat{c}, \hat{e}$, that is, $\vec{F}$ satisfies the law $\left[x_{1}^{\hat{e}}, \ldots, x_{\hat{c}}^{\hat{e}}\right] \equiv 1$. Lifting this to $F$, we have

$$
\left[x_{1}^{\hat{e}}, \ldots, x_{\hat{c}}^{\hat{e}}\right]=w_{1} d \text { in } F,
$$

for some $w_{1} \in W, d \in F^{(2)}$. Since $d \in F^{(2)}$ it may be expressed as a product $d_{1} \cdots d_{t}$ of commutators of the form $d_{i}=\left[\alpha_{i}, \beta_{i}\right]$ where $\alpha_{i}, \beta_{i}$ are commutators of weights $\geq 2$ with all entries from among $x_{1}^{ \pm 1}, \ldots, x_{\hat{c}}^{ \pm 1}$. (If any $d_{i}$ had entries $x_{l}^{ \pm 1}$ with $l>\hat{c}$, we could eliminate these $d_{i}$ by applying to (12) the substitution $x_{i} \rightarrow x_{i}(i=1, \ldots, \hat{c})$, $x_{l} \rightarrow 1(l>\hat{c})$.) 
We now show that there is in fact an equation of the form (12) in $F$ with $d$ a product of commutators (as before) each of which involves all of $x_{1}^{ \pm 1}, \ldots, x_{\hat{c}}^{ \pm 1}$ as entries. Suppose for instance that some of the $d_{i}$ in $d=d_{1} \cdots d_{t}$ do not involve $x_{1}$. Setting $x_{1} \rightarrow 1$ in (12) then yields $1=w_{2} d_{i_{1}} \cdots d_{i_{l}}$ in $F$, where $w_{2}$ results from $w_{1}$, and the $d_{i_{j}}$ are, in the same order as before, just those commutator factors of $d$ not involving $x_{1}^{ \pm 1}$. Hence $d_{i_{1}} \cdots d_{i_{1}} \in W$, whence the original right-hand side of (12) can be rewritten as

$$
\begin{aligned}
w_{1} d & =w_{1} d_{i_{1}} \cdots d_{i_{i}}\left(d_{i_{1}} d_{i_{2}} \cdots d_{i_{l}}\right)^{-1} d_{1} \cdots d_{t} \\
& =w_{3}\left(d_{i_{1}} d_{i_{2}} \cdots d_{i_{i}}\right)^{-1} d_{1} \cdots d_{t},
\end{aligned}
$$

where $w_{3}:=w_{1} d_{i_{1}} \cdots d_{i_{1}} \in W$. It is now easy to see that $\left(d_{i_{1}} \cdots d_{i_{i}}\right)^{-1} d_{1} \cdots d_{t}$ can be rewritten as a product of commutators of the required form all involving $x_{1}^{ \pm 1}$. One now continues by treating $x_{2}$ in the same way, and so on.

We now apply this lemma to the situation of our finite $p$-group $G$ (with $p$ 'sufficiently large', satisfying the law $w \equiv 1$. By this lemma $w \equiv 1$ has a consequence of the form

$$
\left[x_{1}^{\hat{e}}, \ldots, x_{\hat{\boldsymbol{c}}}^{\hat{e}}\right] \equiv \rho,
$$

where $\rho \in F^{(2)}$ and is a product of commutators in $x_{1}, \ldots, x_{\hat{c}}$, each involving all of $x_{1}, \ldots, x_{\hat{c}}$. By collecting those of these commutators involving each of $x_{1}, \ldots, x_{\hat{c}}$ exactly once, we obtain $\rho=\rho_{1} \rho_{2}$ where now $\rho_{1}$ is a product of commutators from $F^{(2)}$ involving each $x_{i}$ exactly once, and $\rho_{2}$ is a product of commutators from $F^{(2)}$ of weights $>\hat{c}$. Hence on making the substitution $x_{1} \rightarrow x, x_{2}, \ldots, x_{\hat{c}} \rightarrow y$, the word $\rho_{1}\left(x_{1}, \ldots, x_{\hat{c}}\right)$ becomes $\rho_{1}(x, y, \ldots, y)=1$, since each commutator factor of $\rho_{1}\left(x_{1}, \ldots, x_{\hat{c}}\right)$ is of the form $[\mu, \nu]$ where $\mu, \nu$ are commutators in disjoint sets of elements from $\left\{x_{1}, \ldots, x_{\hat{c}}\right\}$. Hence we have a law of the following form holding in $G$ :

$$
[x^{\hat{e}}, \underbrace{y^{\hat{e}}, \ldots, y^{\hat{e}}}_{\hat{c}-1}] \equiv \rho_{2}(x, y, \ldots, y),
$$

where $\rho_{2}$ is a product of commutators from $F^{(2)}$ in $x$ and $y$ of weights $>\hat{c}$.

We now impose a preliminary lower bound on $p$, namely:

$$
p>\hat{e} .
$$

From this assumption and the law (13) which our $p$-group $G$ satisfies, it follows that $G$ satisfies a law of the form

$$
[x, \underbrace{y, \ldots, y}_{\hat{i}-1}] \equiv \rho_{3}(x, y)
$$


where $\rho_{3}$ is a product of commutators in $x$ and $y$ (from $F^{(2)}$ ) of weights $>\hat{c}$.

Write $L=L(G)$ for the Lie ring determined in the usual way by the lower central series $G=G_{0}>G_{1}>\cdots>G_{k}=\{1\}$ of $G$, that is, with additive structure given by that of the abelian group

$$
\left(G_{0} / G_{1}\right) \oplus\left(G_{1} / G_{2}\right) \oplus \cdots \oplus\left(G_{k-1} / G_{k}\right)
$$

(written additively), and the Lie multiplication by the rule $\left[a G_{i}, b G_{j}\right]:=[a, b] G_{i+j+1}$, for $a \in G_{i-1}, b \in G_{j-1}$, extended linearly.

Since in the law (14) the left-hand side has weight $\hat{c}$ while wt $\rho_{3}>\hat{c}$, it follows (as in the proof of [22, Lemma 6], by replacing $y$ by $y_{1} \cdots y_{\hat{c}-1}$ in (14) and expanding) that $L(G)$ satisfies the 'linearized Engel law'

$$
\sum_{\sigma \in S_{i-1}}\left[x, y_{\sigma(1)}, \ldots, y_{\sigma(\hat{c}-1)}\right]=0 .
$$

The argument now continues somewhat as in [2, page 517]. Setting $y=y_{1}=\cdots=$ $y_{\hat{c}-1}$ in $(15)$ we infer that the subring $(\hat{c}-1) ! L$ satisfies the $(\hat{c}-1)$-Engel condition $\left[x, \hat{c}_{-1} y\right]=0$. Hence by [2, Corollary 4$]$ (a consequence of Zelmanov's deep results on the Engel condition in Lie algebras-see [21]) there exist positive integers $e_{3}, c_{3}$ depending on $\hat{c}$ such that

$$
e_{3}((\hat{c}-1) ! L)^{c_{3}}=0
$$

that is

$$
e_{3}[(\hat{c}-1) !]^{c_{3}} L^{c_{3}}=e_{4} L^{c_{3}}=0,
$$

where we have written $e_{4}:=e_{3}[(\hat{c}-1) !]^{c_{3}}$. Back in the group $G$, (16) yields

$$
\left(\gamma_{c_{3}}(G)\right)^{e_{4}} \leq \gamma_{c_{3}+1}(G) \text {. }
$$

We now impose a further lower bound on $p$, assuming in addition that

$$
p>e_{4} \text {. }
$$

From this assumption it follows that $\left(\gamma_{c_{3}}(G)\right)^{e_{4}}=\gamma_{c_{3}}(G)$, and then from (17) we deduce that $G$ has class $\leq c_{3}$.

Hence provided $p>\max \left\{\hat{e}, e_{4}\right\}$, the $p$-group $G$ satisfying the law $w \equiv 1$ will have nilpotency class $\leq c_{3}$ where $\hat{e}, e_{4}, c_{3}$ all depend only on the word $w$. Much as before, if we now take $T$ to be the maximum of all such $\hat{e}, e_{4}$ obtained as above for all the (finitely many) words of length $\leq N$ satisfying the assumption of Theorem $\mathrm{A}$, then $T=T(N)$, that is, depends only on $N$, and we conclude that:

If $G$ is any $p$-group with $p>T(N)$ satisfying the law $w \equiv 1$, then there is a positive integer $c_{4}(N)$ also depending only on $N$ (namely the largest of all the $c_{3}$ obtained as above for all words $w$ of length $\leq N$ satisfying the assumption of Theorem A) such that $G$ has nilpotency class $\leq c_{4}(N)$.

Thus the proof in Subcase 2.3.1 is complete. 
2.3.2. Subcase: $p \leq T(N)$. We are now reduced to the situation where $G$ is a finite $p$-group satisfying the law $w \equiv 1$, with $p \leq T(N)$.

We shall need the following two lemmas.

LEMMA 4 (Compare [20, Proof of Lemma 3.2, Case 2]). Let $V$ be a vector space over $\mathbb{Z}_{p}$ and $T$ an invertible linear transformation of $V$ whose order $s$ is a power of $p$. Suppose that there exist integers $m, q>0$ such that $\left(T^{m}-1\right)^{q}=0$. Then the order $s$ of $T$ satisfies the inequality $s<m p q$.

PROOF. Write $s=p^{r}, r \geq 1$. (If $s=1$ the desired inequality holds trivially.) Since $T$ satisfies the polynomial $z^{p^{r}}-1=(z-1)^{p^{r}}$ in $\mathbb{Z}_{p}[z]$, the minimal polynomial of $T$ is a power of $(z-1)$, say $(z-1)^{l}$. From $l \leq p^{r-1}$ it would follow that $T^{p^{r-1}}-1=(T-1)^{p^{r-1}}=0$, contradicting the assumption that $T$ has order $p^{r}$. Hence $p^{r} \geq l>p^{r-1}$. Since by hypothesis $T$ also satisfies $\left(z^{m}-1\right)^{q}=0$ we must have $m q \geq l>p^{r-1}$. Hence $m q p>p^{r}=s$, as required.

REMARK. In Case 2 of [20, Lemma 3.2] slightly more is claimed on the basis of the assumption $\left(T^{m}-1\right)^{q}=0$, namely that $s \leq 2 m q$. However the example of $V:=\mathbb{Z}_{p} \oplus \mathbb{Z}_{p}, T=\left[\begin{array}{ll}1 & 1 \\ 0 & 1\end{array}\right]$, shows this to be false in general, since here $T$ has order $p$, yet $(T-1)^{2}=0$, so that taking $m=1, q=2$, we have $2 m q \succeq p=s$ for primes $p>3$.

LEMMA 5 (Compare [19, Lemma 3.3]). Let $f(z)$ be a polynomial over $\mathbb{Z}$ of degree $d>0$ with non-zero constant term, and let $J$ be the ideal of $\mathbb{Z}[z]$ generated by $f(z), f\left(z^{2}\right), \ldots, f\left(z^{d+1}\right)$. There exist positive integers $q, m$ bounded above by $a$ function of d only, and a positive integer $r$ determined by $f$, such that $r\left(z^{m}-1\right)^{q} \in J$.

Proof. The ideal of $\mathbb{Q}[z]$ corresponding to $J$ is $\mathbb{Q} \otimes J=: I$, say. Applying Lemma 2 of Section 2.2 to this ideal, with $K=\mathbb{Q}$, we infer the existence of $d$ bounded positive integers $m, q$ such that $\left(z^{m}-1\right)^{q} \in I$. Hence there exists a positive integer $r$ such that $r\left(z^{m}-1\right)^{q} \in J$. (Clearly $r$-as well as $m$ and $q$-is determined by the polynomial $f(z)$. However whether $r$ is bounded above by a function of $d$ alone is not clear.)

We are now ready for the proof of Theorem $\mathrm{A}$ in the situation of a finite $p$-group $G$ with $p \leq T(N)$ satisfying the law $w \equiv 1$. The argument largely follows that of Shalev [20], with some adjustments.

Let $H / K$ be any elementary abelian normal section of $G$, that is, $H, K \unlhd G, K<$ $H, H / K$ an elementary abelian $p$-group, and let $g \in G$ be arbitrary. As before we denote by $T_{g}$ the linear transformation of $H / K$ considered as a vector space over $\mathbb{Z}_{p}$, induced by conjugation by $g$. Since $G$ satisfies $w \equiv 1$, by Lemma 2 of Section 2.2 
and the argumentation just preceding and following that lemma, there exist positive integers $m, q$ determined by $w$ alone, such that

$$
\left(T_{g}^{m}-1\right)^{q}=0 .
$$

Applying Lemma 4 above, we infer that the order $s$ ( $=p^{r}$ say) of $T_{g}$ satisfies the inequality $s<m q p$. Since in the present subcase we are assuming $p \leq T(N)$, it follows that for every $g \in G$ the order $s$ of $T_{g}$ is bounded above by a number $D$, say, depending only on the word $w$. Hence $G^{D !}$ centralizes every elementary abelian normal section of $G$. It is not difficult to verify (or see [20, Lemma 4.1]) that therefore $P:=G^{D !}$ (or $P:=G^{2 D !}$ if $p=2$ ) is a powerful $p$-group, that is, $P^{p} \geq[P, P]$ (or $P^{4} \geq[P, P]$ if $p=2$ ).

Now let $A=H_{1} / K_{1}$ be any abelian (not necessarily elementary abelian) normal section of $G$ and again let $g \in G$ be arbitrary. Since the group $\left\langle g K_{1}, A\right\rangle$ is a cyclic extension of the abelian group $A$ and satisfies the law $w \equiv 1$, it follows as in Section 2.2 (see equation (8)) that

$$
a\left(a^{s_{1}}\right)^{\bar{g}^{t_{1}}} \cdots\left(a^{s_{k}}\right)^{\bar{g}^{-t_{k}}}=1 \text { for all } a \in A, l=1,2, \ldots,
$$

where $\bar{g}:=g K_{1}$. Write $\alpha_{g}$ for the automorphism of $A$ induced by conjugation by $g$. Then (compare (9)) the automorphisms $\alpha_{g}^{-l}, l=1,2, \ldots$, all satisfy (in End $A$ with $A$ written additively) the non-constant polynomial of positive degree given by

$$
f(z)=1+s_{1} z^{-t_{1}}+s_{2} z^{-t_{2}}+\cdots+s_{k} z^{-t_{k}} \in \mathbb{Z}[z] .
$$

Writing $J$ for the ideal of $\mathbb{Z}[z]$ generated by all polynomials $h(z)$ such that $h\left(\alpha_{g}^{-1}\right)=0$, we infer from Lemma 5 above the existence of positive integers $r, m, q$ depending on $f(z)$, and therefore ultimately on $w$ only, such that

$$
r\left(\alpha_{g}^{m}-1\right)^{q}=0 \text { in End } A .
$$

Write $e_{5}:=D ! m$ and consider $Q:=G^{e s}$. Since $G^{D ! m} \leq\left(G^{D !}\right)^{m}=P^{m}$, it follows that $Q \leq P^{m}$. Since $P$ is powerful, we have by [10, Propositions 1.7 and 4.1.7] that every element of the group $P^{m}$, and therefore every element of $Q$, is of the form $g^{m}$ for some $g \in P$. Hence for every element $h \in Q$ we have in view of (18)

$$
r\left(\alpha_{h}-1\right)^{q}=0 \text { in End } A .
$$

Since $A$ is a $p$-group it follows that in fact

$$
p^{s}\left(\alpha_{h}-1\right)^{q}=0 \text { in End } A,
$$

where $p^{s}$ is the largest power of $p$ dividing $r$. 
Consider the series $A>p A>p^{2} A>\cdots>p^{\prime} A=0$ for $A$ (written additively). Since each factor $p^{i} A / p^{i+1} A$ is elementary abelian and therefore centralized by $P$ and hence by $Q(\leq P)$, we have for all $h \in Q$

$$
\left(\alpha_{h}-1\right) p^{i} A \leq p^{i+1} A, \quad i=0,1, \ldots, t-1 .
$$

Thus by (19) we have $\left(\alpha_{h}-1\right)^{q+s}=0$ in End $A$ for all $h \in Q$. Translated into multiplicative notation this becomes

$$
[A, q+s]=\{1\} \text { for all } h \in Q \text {. }
$$

Write $c^{\prime}$ for the nilpotency class of $Q$, and $c^{\prime \prime}:=\left\lceil\left(c^{\prime}+1\right) / 2\right\rceil$. Then $\gamma_{c^{\prime \prime}}(Q)$ is a fully invariant abelian subgroup of $Q=G^{e_{5}}$ and hence of $G$, so that by (20)

$$
\left[\gamma_{c^{\prime \prime}}(Q),{ }_{q+s} h\right]=\{1\} \quad \text { for all } h \in Q \text {. }
$$

By [20, Proposition D] it follows that

$$
[\gamma_{c^{\prime \prime}}(Q), \underbrace{Q, Q, \ldots, Q]}_{\beta}]=\{1\}
$$

for some $\beta=\beta(q+s, d(Q))$ where $d(Q)$ is the least number of generators of $Q$. Hence $c^{\prime} \leq \beta+\left\lceil\left(c^{\prime}+1\right) / 2\right\rceil$, yielding $c^{\prime} \leq \beta+\left(c^{\prime}+2\right) / 2$, whence $c^{\prime} \leq 2 \beta+2$.

Now since $Q=G^{e_{5}}$, the quotient $G / Q$ has exponent bounded above by $e_{5}$. Hence by Zelmanov's solution of the Restricted Burnside Problem [24, 23], $|G / Q|$ is bounded above by a function of $e_{5}$ and $d(G)$. Hence $d(Q)$ is also so bounded, whence $\beta$ and therefore also the class $c^{\prime}$ of $Q$ are so bounded.

We separate out part of what we have so far proved (for use in a slightly different context below) as a

PROPOSITION (Compare [20, Proposition 5.3]). Given any $d>0$, if $G$ is any finite d-generator $p$-group with $p \leq T(N)$, satisfying the law $w \equiv 1$, then there exist positive integers $e_{5}=e_{5}(N)$ and $c^{\prime}=c^{\prime}(N, d)$ (where $N$ is the length of $w$ ) such that

$$
\gamma_{c^{\prime}+1}\left(G^{e^{s}}\right)=\{1\}
$$

(Note that $G^{e_{5}}=: Q$ above. Note also that from the preceding argument we may immediately infer only the dependence of $e_{5}$ and $c^{\prime}$ on the word $w$ rather than just its length. However by the usual argument (see earlier), involving possible replacement of $e_{5}$ and $c^{\prime}$ by larger numbers, we may in fact assume dependence only on $N$-as well as $d$ in the case of $c^{\prime}$.) 
We now return to the powerful $p$-group $P\left(:=G^{D !}\right)$. Let $g_{1}, g_{2}$ be any elements of $P$. Applying the above proposition to $P_{0}:=\left\langle g_{1}, g_{2}\right\rangle$ we have, since $P_{0}$ is 2-generator, that

$$
\gamma_{\left(c^{\prime}(N, 2)+1\right)}\left(P_{0}^{e_{5}}\right)=\{1\}
$$

Writing $c_{5}(N):=c^{\prime}(N, 2)$, it follows from (21) in particular that $\left[g_{1}^{e_{5}},{ }_{c_{5}} g_{2}^{e_{5}}\right]=1$ for all $g_{1}, g_{2} \in P$, that is, $P$ satisfies the law

$$
\left[x^{e_{s}}, c_{s} y^{e_{s}}\right]=1 \text {, }
$$

where $e_{5}, c_{5}$ depend only on $N$.

The proof now continues as in [2, page 515], starting from the second displayed formula on that page. (Note that the first displayed formula there is incorrect.) That argument starts with a powerful $p$-group satisfying the law $\left[x^{e_{1}}, c_{1} y^{e_{1}}\right] \equiv 1$ for some $e_{1}, c_{1}$, and concludes (at the top of p.518) that there exist numbers $e_{3}, c_{3}$ depending only on $e_{1}, c_{1}$ such that the original powerful $p$-group belongs to $\mathfrak{N}_{e_{3}} \hat{\mathfrak{B}}_{e_{3}}$. Translating this into our present notation, we infer the existence of numbers $e_{6}, c_{6}$ depending only on $e_{5}, c_{5}$ such that $P \in \mathfrak{N}_{c_{6}} \hat{\mathfrak{B}}_{e_{6}}$. Then since $P=G^{D !}$ we have the desired conclusion for $G$, completing the proof in Subcase 2.3.2, and with that the proof of Theorem A for finite groups.

\section{Completion of the proof of Theorem A}

We now show that any finitely generated soluble-by-finite group $G$ satisfying the law $w \equiv 1$ lies in the variety $\mathfrak{N}_{c} \mathfrak{B}_{e}$ for some $c=c(N), e=e(N)$ depending only on the length $N$ of $w$. In view of this dependence of $c, e$ only on $N$, Theorem A then follows immediately.

The argument will follow that of [2] beginning on page 518, adapted to our present situation. (In particular the context in [2, page 518 et seqq.] of a generating subsemigroup $S$ of $G$ satisfying a semigroup law will no longer be relevant. However the proof given there of Theorem $D$ to the effect that if $S$ satisfies a semigroup law then that law actually holds in $G$ (and furthermore $\left.G \in \mathfrak{N}_{c(N)} \hat{\mathfrak{B}}_{e(N)}\right)$ remains valid.)

With $G$, as above, any finitely generated soluble-by-finite group satisfying the law $w \equiv 1$, suppose that $m$ is such that $G^{m}$ is soluble. Observe first that by a well-known result of Hall (see for example [17, 15.4.1, page 453]) the quotient

$$
\bar{G}:=G /\left[\gamma_{k}\left(G^{m}\right), \gamma_{k}\left(G^{m}\right)\right]
$$

is residually finite for every $k$ since it is abelian-by-nilpotent-by-finite. Hence there is a chain $\bar{G} \geq \bar{G}_{1} \geq \bar{G}_{2} \geq \cdots$ of normal subgroups of $\bar{G}$ such that $\left|\bar{G}: \bar{G}_{i}\right|<\infty$ 
and $\cap \bar{G}_{i}=\{1\}$. Since $\bar{G} / \bar{G}_{i}$ is finite and satisfies the law $w \equiv 1$, we have by the finite case of the theorem, already established, that $\bar{G} / \bar{G}_{i} \in \mathfrak{N}_{c} \hat{\mathfrak{B}}_{e}$ for some $c=c(N)$, $e=e(N)$ depending only on the length $N$ of $w$. Since $\bar{G}$ is a subcartesian product of the $\bar{G} / \bar{G}_{i}$, it follows that $\bar{G} \in \mathfrak{N}_{c} \hat{\mathfrak{B}}_{e}$, or, equivalently,

$$
\gamma_{c}\left(G^{e}\right) \leq\left[\gamma_{k}\left(G^{m}\right), \gamma_{k}\left(G^{m}\right)\right] \text { for all } k
$$

Consider now the descending chain $G=N_{0} \geq N_{1} \geq N_{2} \geq \cdots$ of subgroups of finite index in $G$, defined inductively by

$$
N_{1}:=G^{m e}, \quad N_{i+1}:=N_{i}^{e}, \quad \text { for } i \geq 1 .
$$

Since the $N_{i}$ are all finitely generated subgroups of $G$, the argument leading to (23) applies to each of them, so that

$$
\gamma_{c}\left(N_{i}^{e}\right) \leq\left[\gamma_{k}\left(N_{i}\right), \gamma_{k}\left(N_{i}\right)\right] \text { for all } i, k
$$

that is,

$$
\gamma_{c}\left(N_{i+1}\right) \leq\left[\gamma_{k}\left(N_{i}\right), \gamma_{k}\left(N_{i}\right)\right] \text { for all } i, k
$$

Hence writing $l$ for the solubility length of $G^{m}$, we have in particular

$$
\gamma_{c}\left(N_{l+1}\right) \leq \gamma_{c}\left(N_{l}\right)^{(1)} \leq \gamma_{c}\left(N_{l-1}\right)^{(2)} \leq \cdots \leq \gamma_{c}\left(N_{1}\right)^{(l)}=\{1\}
$$

where the equality follows from the fact that $N_{1}=G^{m e} \leq G^{m}$ and $G^{m}$ is soluble of length $l$. Hence $G$ is a finite extension of a group of class $\leq c$, namely $N_{l+1}$, and is therefore residually finite, whence we infer, as earlier, that $G \in \mathfrak{N}_{c} \hat{\mathfrak{B}}_{e}$.

\section{References}

[1] S. Black, 'Which words spell "almost nilpotent" ?', preprint, 1997.

[2] R. G. Burns, O. Macedońska and Y. Medvedev, 'Groups satisfying semigroup laws, and nilpotentby-Burnside varieties', J. Algebra 195 (1997), 510-525.

[3] R. G. Burns and Y. Medvedev, 'A note on Engel groups and local nilpotence', J. Austral. Math. Soc. Ser. A 64 (1998), 92-100.

[4] J. R. J. Groves, 'Varieties of soluble groups and a dichotomy of P. Hall', Bull. Austral. Math. Soc. 5 (1971), 391-410.

[5] K. W. Gruenberg, 'Two theorems on Engel groups', Math. Proc. Cambridge Philos. Soc. 49 (1953), 377-380.

[6] B. Huppert, Endliche Gruppen I (Springer, Berlin, 1967).

[7] G. A. Jones, 'Varieties and simple groups', J. Austral. Math. Soc. 17 (1974), 163-173.

[8] M. I. Kargapolov and Ju. I. Merzljakov, Fundamentals of the theory of groups (Springer, Berlin, 1979). 
[9] Y. K. Kim and A. H. Rhemtulla, 'Weak maximality condition and polycyclic groups', Proc. Amer. Math. Soc. 123 (1995), 711-714.

[10] A. Lubotzky and A. Mann, 'Powerful p-groups, I. Finite groups', J. Algebra 105 (1987), 484-505.

[11] A. I. Mal'cev, 'Nilpotent semigroups', Uchen. Zap. Ivanovsk. Ped. Inst. 4 (1953), 107-111.

[12] B. H. Neumann and T. Taylor, 'Subsemigroups of nilpotent groups', Proc. Roy. Soc. Ser. A 274 (1963), $1-4$.

[13] H. Neumann, Varieties of groups (Springer, Berlin, 1967).

[14] A. Yu. Olshanskiǐ and A. Storozhev, 'A group variety defined by a semigroup law', J. Austral. Math. Soc. Ser. A 60 (1996), 255-259.

[15] F. Point, 'Milnor identities', Comm. Algebra 24 (1996), 3725-3744.

[16] D. J. S. Robinson, Finiteness conditions and generalized soluble groups. Part I (Springer, Berlin, 1972).

[17] - A course in the theory of groups (Springer, Berlin, 1982).

[18] J. M. Rosenblatt, 'Invariant measures and growth conditions', Trans. Amer. Math. Soc. 193 (1974), 33-53.

[19] J. F. Semple and A. Shalev, 'Combinatorial conditions in residually finite groups, I', J. Algebra 157 (1993), 43-50.

[20] A. Shalev, 'Combinatorial conditions in residually finite groups, II', J. ALgebra 157 (1993), 51-62.

[21] E. I. Zelmanov, 'Engel Lie algebras', Dokl. Akad. Nauk SSSR 292 (1987), 265-268.

[22] _ - 'On some problems of group theory and Lie algebras', Math. USSR-Sbornik 66 (1990), 159-168.

[23] _ 'The solution of the restricted Burnside problem for 2-groups', Mat. Sb. 182 (1991), $568-592$.

[24] — 'The solution of the restricted Burnside problem for groups of odd exponent', Math. USSR-Izv. 36 (1991), 41-60.

Department of Mathematics and Statistics

Bank of Montreal

York University

Toronto, Ontario

Toronto, Ontario

Canada

Canada M3J 1P3

e-mail: rburns@pascal.math.yorku.ca 